\title{
Null scrolls with prescribed curvatures in Lorentz-Minkowski 3-space
}

\author{
Željka Milin Šipuš, Ljiljana Primorac Gajčić and Ivana Protrka
}

\begin{abstract}
In Lorentz-Minkowski 3-space, null scrolls are ruled surfaces with a null base curve and null rulings. Their mean, as well as their Gaussian curvature, depends only on a parameter of a base curve. In the present paper, we obtain the first-order nonlinear differential equation (Riccati equation) which relates curvatures of a base curve to curvatures of a null scroll. Conditioned by this equation, we can determine a family of null scrolls with a given null base curve and prescribed curvatures, in particular, a family of minimal and constant mean curvature null scrolls.
\end{abstract}

\section{Introduction}

The Lorentz-Minkowski space, usually considered as the ambient space for Einstein's theory of relativity, also attracts interest of mathematicians, due to its pseudo-metric which provides more variations in geometrical notions than Euclidean space. For example, contrary to the theory of curves in Euclidean space, in Lorentz-Minkowski space a proper frame for a null curve is not unique, so a null curve must be given together with a frame, [5]. Furthermore, $B$-scrolls, that is, ruled surfaces with a null base curve and null rulings determined by binormals of a base curve, include examples of surfaces that have no Euclidean counterparts, characterized by the property that their shape operator is not diagonalizable, [7].

Key Words: Null scroll, prescribed curvature, Lorentz-Minkowski space.

2010 Mathematics Subject Classification: Primary 53A10; Secondary 53C50.

Received: 22.01.2020

Accepted: 29.02.2020 
In the present paper, we deal with ruled surfaces with a null base curve and null rulings, the so called null scrolls, and show that for a given base curve and prescribed mean, resp. Gaussian, curvature, we can construct a family of null scrolls of a given curvature.

The problem of finding surfaces with prescribed Gaussian $K$ or mean curvature $H$ is a well known problem of classical differential geometry. In general, it is directly related to the study of non-linear partial differential equations, in particular Monge-Ampère equation. For example, in [3], author studied surfaces parametrized by the isothermal coordinates and considered a problem of solving semilinear elliptic partial differential equation, while in [13], author considered curvature of a surface as a function of the asymptotic coordinates and gave conditions on Gaussian curvature to describe a surface. However, the generality of the problem is reduced in study of e. g. surfaces with constant curvature $K$ or $H$, Weingarten surfaces having functional relationship between $K$ and $H$, or of special classes of surfaces, in particular, rotational and helicoidal surfaces.

In [8], author solved the non-linear ordinary differential equation to obtain the generating curve of the rotational surface with given mean curvature. Analogous approach was adopted for solving the problem of given constant mean, respectively Gaussian curvature, for the helicoidal surfaces, [4]. Differential equations that determine the generating curve of the helicoidal surface with given mean, respectively Gaussian curvature, were presented in [1]. The analogous problems were considered also in the Lorentz-Minkowski space, $[2,6,9,10]$.

In Lorentz-Minkowski space, null scrolls are examples of totally quasi-umbilical surfaces, so for their mean curvature $H$ and Gaussian curvature $K$ the relation $H^{2}-K=0$ holds, however their shape operator need not be diagonalizable. If the mean curvature of a null scroll is prescribed, then the Gaussian curvature is also given, and vice versa. In [11], we showed that if we introduce a null frame for a base curve of a null scroll which is related to its rulings, then its mean curvature is determined by the curvature of a base curve. If an arbitrary null curve is given (assumed to be parametrized by the pseudo-arc parameter), it is of interest to find all null scrolls (assumed to be parametrized by arbitrary parameters) with the given null curve as a base curve and prescribed curvatures. In the analysis that follows we establish relations between the lightlike curvature of a given null base curve (which determines a curve uniquely, up to a position in space), and the curvatures of a null scroll. Such relations allow us to construct a family of null scrolls with a given base curve and prescribed curvatures. In particular, for a given null curve, we obtain also a family of minimal null scrolls with the same base curve. 


\section{Preliminaries}

The 3-dimensional Lorentz-Minkowski space, denoted by $\mathbf{R}_{1}^{3}$, is a real affine space whose underlying vector space $\mathbf{R}^{3}$ is endowed with the pseudo-scalar product defined by

$$
x \cdot y=x_{1} y_{1}+x_{2} y_{2}-x_{3} y_{3},
$$

where $x=\left(x_{1}, x_{2}, x_{3}\right)$ and $y=\left(y_{1}, y_{2}, y_{3}\right)$. A vector $x \in \mathbf{R}_{1}^{3}$ is called spacelike if $x \cdot x>0$ or $x=0$, timelike if $x \cdot x<0$ and null (or lightlike) if $x \cdot x=0$ and $x \neq 0$. The pseudo-norm of a vector $x$ is defined as the real number $\|x\|=\sqrt{|x \cdot x|} \geq 0$. The causal character of a regular curve $c: I \rightarrow \mathbf{R}_{1}^{3}$ is determined by the causal character of its velocity vector $c^{\prime}(u)$. We can frame every null curve $c(u)$ with a proper null frame $L(u)=(A(u), B(u), C(u))$ along $c$, where $c^{\prime}(u)=k_{0} \cdot A(u), k_{0}>0$ and the following holds

$$
A \times B=C, A \times C=-A, B \times C=B .
$$

Vector fields $A, B, C$ satisfy analogues of the Frenet formulas

$$
A^{\prime}=k_{1} A+k_{2} C, B^{\prime}=-k_{1} B+k_{3} C, C^{\prime}=-k_{3} A-k_{2} B,
$$

where the functions $k_{i}, i=1,2,3$ are called the curvature functions of a curve $c$ with respect to a frame $L$. Such null frame is not unique, so a null curve and a frame must be given together, [7]. Furthermore, every null curve $c$ satisfying $c^{\prime \prime} \cdot c^{\prime \prime}>0$ (hence not a line), can be reparametrized by a new parameter $u=u(s)$, called the pseudo-arc parameter, such that $c_{s s} \cdot c_{s s}=1$, and can be framed by $L(s)=(A(s), B(s), C(s))$, whereby vector fields satisfy formulas

$$
A^{\prime}=C, B^{\prime}=k_{L} C, C^{\prime}=-k_{L} A-B .
$$

The function $k_{L}$ defined by $k_{L}=\frac{1}{2} c^{\prime \prime \prime} \cdot c^{\prime \prime \prime}$ is called the lightlike curvature. It determines a null curve uniquely, up to a position in space. Moreover, (2) implies that the frame $L(s)=(A(s), B(s), C(s))$ is generated by the vector fields related to the curve $c$ as

$$
A=c^{\prime}, \quad B=-k_{L} c^{\prime}-c^{\prime \prime \prime}, C=c^{\prime \prime} .
$$

A surface $S$ in $\mathbb{R}_{1}^{3}$ is said to be spacelike, timelike or lightlike if the induced metric on $S$ is positive definite, indefinite or degenerate, respectively. A ruled surface is a surface traced out by a straight line moving along a curve $c$ and therefore it admits a local parametrization of the form

$$
f(u, v)=c(u)+v e(u), u \in I \subset \mathbb{R}, v \in \mathbb{R} .
$$


A curve $c$ is the base curve (the generating curve) and straight lines with directions $e(u)$ are the rulings of a surface. In $\mathbb{R}_{1}^{3}$ ruled surfaces are classified with respect to the causal character of their base curve and their rulings (spacelike, timelike or null). It can be shown that, after an appropriate change of the base curve, classes can locally be represented either by having the base curve and rulings non-null and linearly independent or null and linearly independent. We refer to the latter as null scrolls. $B$-scrolls are null scrolls with a base curve parametrized by the so-called distinguished parameter, that is, framed by $L=(A, B, C)$ that satisfies (1) with $k_{1} \equiv 0$, and rulings determined by the binormals $B$ of a base curve. It is always possible to reparametrize a null base curve by a distinguished parameter, [7].

In [11] it was shown that if the base curve of a null scroll is framed by a null frame $L=(A, B, C)$ whose vector field $B$ is collinear to the rulings of a null scroll, then the mean curvature $H$ of a null scroll is given by $H=k_{3}$, where $k_{3}$ is the curvature function of $c$ with respect to $L$. Following this approach, in this paper we establish relations which will be used in defining null scrolls with a given base curve and prescribed curvatures. Results are presented in the next section.

\section{$3 \quad$ Null scrolls with prescribed curvatures}

Let $S$ be a null scroll given by the parametrization (4), where $u$ is the pseudoarc parameter of a null base curve $c$ and $L(u)=(A(u), B(u), C(u))$ its null frame of the form (2) with the lightlike curvature $k_{L}$. Since the rulings $e$ are null, by rescaling them, without loss of generality, we can assume $c^{\prime} \cdot e=1$. If we introduce another null frame for the base curve, related to the rulings of the null scroll, curvatures of the base curve are given by the following lemma.

Lemma 1. Let a base curve $c$ of a null scroll with the parametrization (4), be parametrized by the pseudo-arc parameter. If we frame a curve $c$ with the null frame $\left(c^{\prime}, e, c^{\prime} \times e\right)$, then the associated curvatures $k_{1}, k_{2}$ and $k_{3}$ are given by

$$
k_{1}=c^{\prime \prime} \cdot e, k_{2}=1, k_{3}=\operatorname{det}\left(c^{\prime}, e, e^{\prime}\right) .
$$

Proof. Formulas for curvatures $k_{1}$ and $k_{3}$ follow from the Frenet formulas (1). The curve $c$ is parametrized by the pseudo-arc parameter, therefore it is also framed by the null frame $L=(A, B, C)$ of the form (2). Using the relation (3), for the curvature $k_{2}$ we obtain

$$
k_{2}=c^{\prime \prime} \cdot\left(c^{\prime} \times e\right)=-e \cdot\left(c^{\prime} \times c^{\prime \prime}\right)=-e \cdot(A \times C)=e \cdot A=e \cdot c^{\prime}=1 .
$$


It is also known ([11]) that the mean curvature $H$ of $S$ satisfies $H(u)=$ $k_{3}(u)$, whereas for the Gaussian curvature $K(u)=k_{3}^{2}(u)$ holds. In order to obtain relation between the curvature of a base curve and the mean curvature of a null scroll, first we establish relations between vector fields of the frames $L$ and $\bar{L}=(\bar{A}(u), \bar{B}(u), \bar{C}(u))=\left(c^{\prime}, e, c^{\prime} \times e\right)$.

Proposition 1. Let $S$ be a null scroll given by the parametrization of the form (4), where $u$ is the pseudo-arc parameter of a null base curve $c$ and $L(u)=(A(u), B(u), C(u))$ its null frame of the form (2). Then for the vector fields of the null frame $\bar{L}(u)=(\bar{A}(u), \bar{B}(u), \bar{C}(u))$ of the form (1) the following relations hold

$$
\bar{A}=A, \quad \bar{B}=\left(k_{1}^{\prime}-k_{3}+k_{L}\right) A+B+k_{1} C, \quad \bar{C}=C-k_{1} A,
$$

where $k_{L}$ is the lightlike curvature, and $k_{1}$ and $k_{3}$ are curvatures of the base curve with respect to $\bar{L}$.

Proof. Obviously, by definition, vector fields $A$ and $\bar{A}$ correspond. By taking derivatives of $\bar{A}$ and $A$ with respect to $u$, since $k_{2}=1$, we obtain $k_{1} \bar{A}+\bar{C}=C$. Therefore $\bar{C}=C-k_{1} A$. Now, by taking derivatives with respect to $u$ of the vector field $\bar{C}$ we get

$$
-k_{3} \bar{A}-\bar{B}=-k_{L} A-B-k_{1}^{\prime} A-k_{1} C,
$$

hence

$$
\bar{B}=\left(k_{1}^{\prime}-k_{3}+k_{L}\right) A+B+k_{1} C .
$$

Now, since $\bar{B}$ is a lightlike vector field, it follows

$$
\bar{B} \cdot \bar{B}=2\left(k_{1}^{\prime}-k_{3}+k_{L}\right)+k_{1}^{2}=0 .
$$

Expression (5), considered as a Riccati ordinary differential equation for the unknown function $k_{1}$, allows us to construct the sought-after rulings $\bar{B}$ from Proposition 1, of a null scroll with prescribed curvatures $H$ and $K$ over a given base curve with the lightlike curvature $k_{L}(u)$. Therefore, by using $(3)$ we have proved the following statement.

Theorem 1. For a given null curve $c: I \rightarrow \mathbf{R}_{1}^{3}$ with the lightlike curvature $k_{L}(u)$, where $u \in I$ is assumed to be its pseudo-arc parameter, a family of null scrolls with a prescribed mean curvature $H(u)=k_{3}(u)$, is given by

$$
\mathcal{N}(c, H)=\left\{c(u)+v\left(\left(k_{1}^{\prime}(u)-H(u)\right) c^{\prime}(u)+k_{1}(u) c^{\prime \prime}(u)-c^{\prime \prime \prime}(u)\right)\right\},
$$

where the function $k_{1}$ is a solution of the ordinary differential equation

$$
2\left(k_{1}^{\prime}-H+k_{L}\right)+k_{1}^{2}=0 .
$$


We can note that, in particular, the family (6) contains a unique $B$-scroll $f(u, v)=c(u)+v B(u)$ over a given null curve $c$ parametrized by the pseudoarc parameter and framed by $L(u)=(A(u), B(u), C(u))$. It satisfies $k_{1} \equiv 0$, and its mean curvature $H$ coincides with the lightlike curvature $k_{L}$ of $c$, in which case the ordinary differential equation (5) holds trivially. Its binormal field is given by $B(u)=-k_{L}(u) c^{\prime}(u)-c^{\prime \prime \prime}(u)$.

Further, we note that general solutions of the Riccati equation (5) can be explicitly obtained only if a particular solution is known. However, in certain special cases (see [12] for more details), general solutions can be obtained analytically. In order to generate examples, we choose the base curve and mean curvature of the null scroll, so that special cases of Riccati equation are obtained.

Example 1. Let $c$ be the null curve

$$
c(u)=\frac{u^{2}}{10}(4 \sin (\log u)-2 \cos (\log u),-4 \cos (\log u)-2 \sin (\log u), 5),
$$

where $\log$ stands for the natural logarithm. The lightlike curvature of $c$ is given by $k_{L}=1 / u^{2}$. A family of null scrolls generated by $c$ and with the mean curvature $H=5 / u^{2}$ is determined by the curvature functions $k_{1}$ given by

$$
k_{1}(u)=-\frac{1}{u}\left(3\left(\frac{2 c_{1}}{c_{1}+u^{3}}-1\right)-1\right), c_{1} \in \mathbb{R} .
$$

The family of null scrolls with the base curve $c$ and given mean curvature $H$ is constructed by the means of formula (6), see Figure 1.
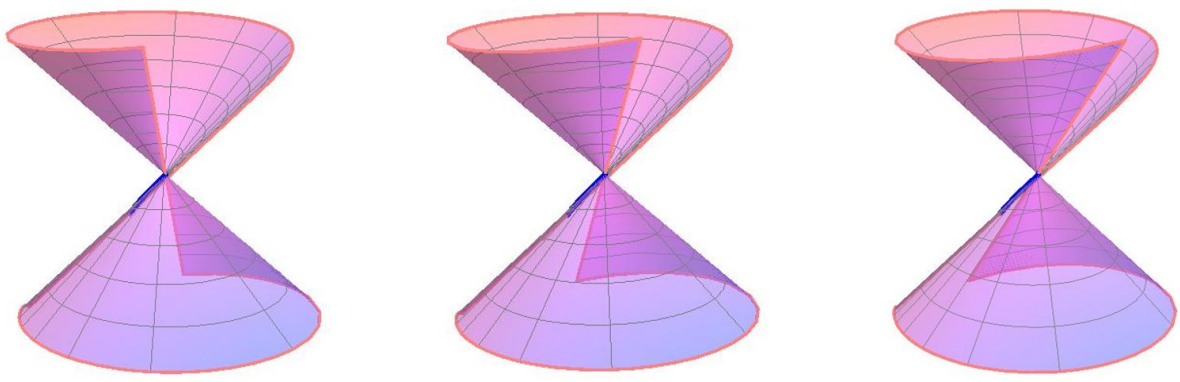

Figure 1: Examples of null scrolls with mean curvature $H=\frac{5}{u^{2}}$ and the base curve $c(u)=\frac{u^{2}}{10}(4 \sin (\log u)-2 \cos (\log u),-4 \cos (\log u)-2 \sin (\log u), 5)$ for different values of parameters $c_{1} \in \mathbb{R}$ 
From the expression (5) we can also obtain characterization of null scrolls of constant curvatures as follows.

Corollary 1. Let $S$ be a null scroll with a base curve parametrized by the pseudo-arc parameter and with the lightlike curvature $k_{L}(u)$. Then $S$ is a null scroll of constant Gaussian and mean curvatures if and only if curvature functions $k_{L}$ and $k_{1}$ (with respect to $\bar{L}=(\bar{A}(u), \bar{B}(u), \bar{C}(u)$ )) satisfy

$$
2\left(k_{1}^{\prime}+k_{L}\right)+k_{1}^{2}=\text { const. }
$$

Example 2. In the Lorentz-Minkowski space we distinguish between three different types of null helices (with respect to its axis of rotation). Their lightlike curvatures are $k_{L}=a^{2} / 2, k_{L}=-a^{2} / 2$ or $k_{L}=0$, and their respective parametrizations

$$
\begin{aligned}
c_{1}(u) & =\left(\frac{1}{a^{2}} \cos (a u), \frac{1}{a^{2}} \sin (a u),-\frac{u}{a}\right), \\
c_{2}(u) & =\left(-\frac{u}{a}, \frac{1}{a^{2}} \cosh (a u), \frac{1}{a^{2}} \sinh (a u)\right), \\
c_{3}(u) & =\left(\frac{u^{3}}{4}-\frac{u}{3}, \frac{u^{2}}{2}, \frac{u^{3}}{4}+\frac{u}{3}\right) .
\end{aligned}
$$

Null scrolls with constant mean curvature $H$ and with null helices $c_{1}, c_{2}, c_{3}$ as base curves are given by (see Figure 2.)

$$
\begin{aligned}
f_{1}(u, v) & =c_{1}(u)+v\left(\left(\frac{-a^{2}+2 H}{1+\cos \left(\sqrt{a^{2}-2 H}\left(u-2 C_{1}\right)\right)}-H\right) c_{1}^{\prime}(u)+\right. \\
& \left.+\sqrt{a^{2}-2 H} \tan \left(\frac{\sqrt{a^{2}-2 H}\left(-u+2 C_{1}\right)}{2}\right) c_{1}^{\prime \prime}(u)-c_{1}^{\prime \prime \prime}(u)\right), \quad C_{1} \in \mathbb{R}, \\
f_{2}(u, v) & =c_{2}(u)+v\left(\left(\frac{a^{2}+2 H}{1+\cos \left(\sqrt{-a^{2}-2 H}\left(u+2 C_{2}\right)\right)}-H\right) c_{2}^{\prime}(u)+\right. \\
& \left.+\sqrt{-a^{2}-2 H} \tan \left(\frac{\sqrt{-a^{2}-2 H}\left(-u-2 C_{2}\right)}{2}\right) c_{2}^{\prime \prime}(u)-c_{2}^{\prime \prime \prime}(u)\right), C_{2} \in \mathbb{R}, \\
f_{3}(u, v) & =c_{3}(u)+v\left(H \operatorname{sech}^{2}\left(\frac{\left.\sqrt{H}\left(u+2 C_{3}\right)\right)}{\sqrt{2}}-H\right) c_{3}^{\prime}(u)+\right. \\
& \left.+\sqrt{2 H} \tanh \left(\frac{\sqrt{2 H} u+2 \sqrt{2 H} C_{3}}{2}\right) c_{3}^{\prime \prime}(u)-c_{3}^{\prime \prime \prime}(u)\right), \quad C_{3} \in \mathbb{R} .
\end{aligned}
$$

In particular, a null scroll is minimal (and therefore flat) if the curvature functions satisfy $2\left(k_{1}^{\prime}+k_{L}\right)+k_{1}^{2}=0$. 

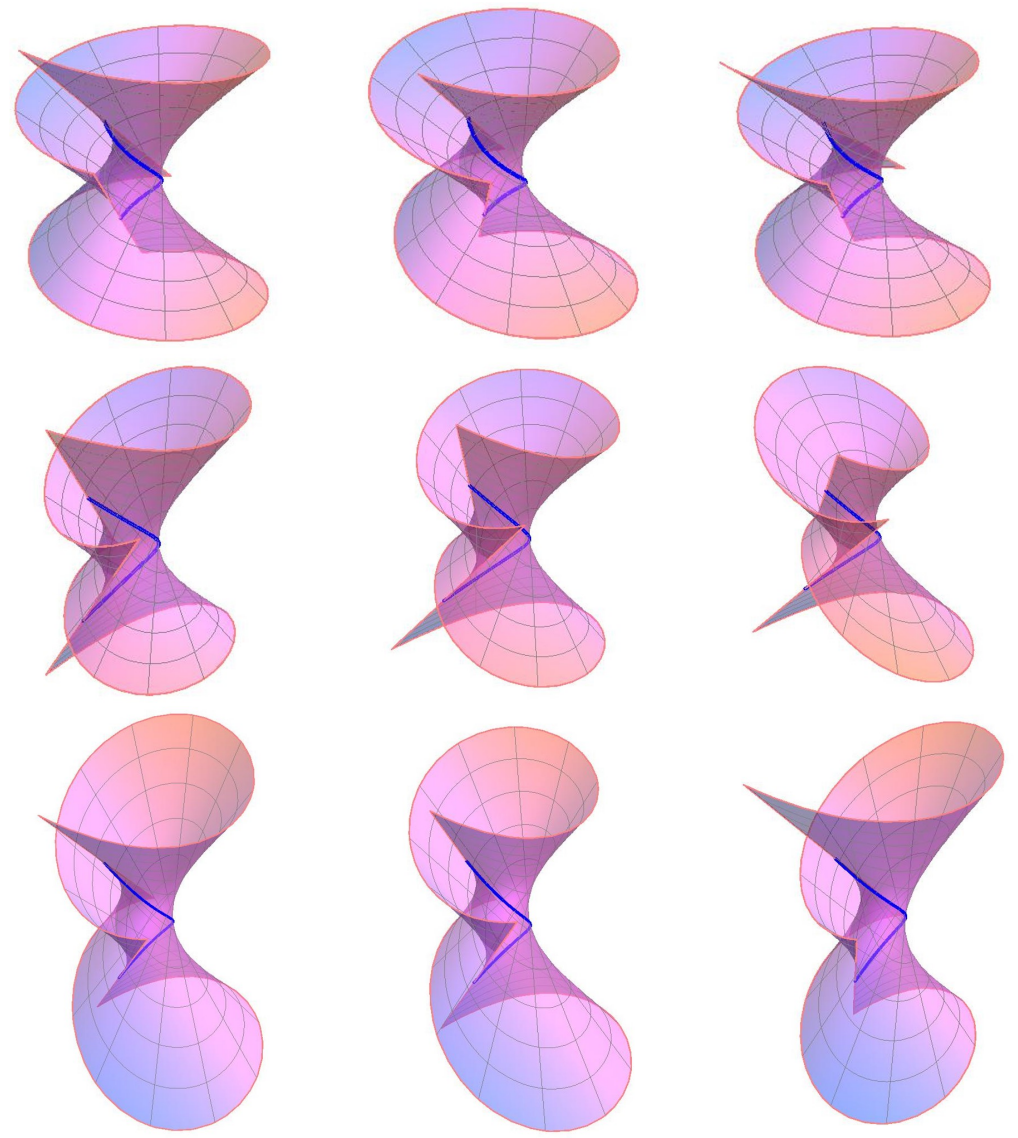

Figure 2: Examples of null scrolls with mean curvature $H=3$ and null helices $c_{1}, c_{2}, c_{3}$ as base curves for different values of parameters $C_{1}, C_{2}, C_{3} \in \mathbb{R}$ and $a=2$

Theorem 2. For every null curve $c(u)$ with the ligthlike curvature $k_{L}(u)$, a family of minimal null scrolls constructed on $c$ is given by

$$
\mathcal{M}(c)=\left\{c(u)+v\left(k_{1}^{\prime}(u) c^{\prime}(u)+k_{1}(u) c^{\prime \prime}(u)-c^{\prime \prime \prime}(u)\right)\right\},
$$

where $k_{1}$ is the solution of the Riccati ordinary differential equation

$$
2\left(k_{1}^{\prime}+k_{L}\right)+k_{1}^{2}=0 .
$$

Example 3. Let c be the null curve $c(u)=\frac{1}{8} u^{2}\left(4 \log u-2,2 \log ^{2} u-2 \log u-1\right.$, 
$\left.2 \log ^{2} u-2 \log u+3\right)$. The lightlike curvature of $c$ is given by $k_{L}(u)=1 /\left(2 u^{2}\right)$. The family of minimal null scrolls generated by $c$ is determined by the curvature functions $k_{1}$ given by the particular solution

$$
k_{1}(u)=\frac{1}{u}\left(\frac{2}{\log u+c_{1}}+1\right), \quad c_{1} \in \mathbb{R} .
$$

See Figure 3. Note that also the function $k_{1}(u)=1 / u$ is the particular solution of the Riccati equation (7).
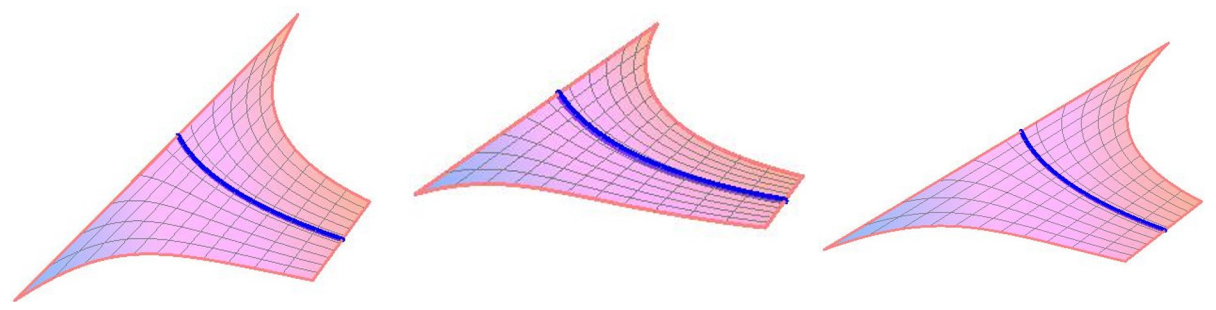

Figure 3: Examples of minimal null scrolls with the base curve $c(u)=\frac{1}{8} u^{2}\left(4 \log (u)-2,2 \log ^{2}(u)-2 \log (u)-1,2 \log ^{2}(u)-2 \log (u)+3\right)$

A parabolic null cylinder (see Figure 4), i. e. helicoidal ruled surface obtained by the moving of a lightlike line around a lightlike axis, is an example of a minimal null scroll. Its parametrization has the form

$$
f(u, v)=\left(b+h\left(\frac{u^{3}}{3}-u\right), h u^{2}, b+h\left(\frac{u^{3}}{3}+u\right)\right)+v(a, 0, a), a>0, b, h \in \mathbb{R} .
$$

The base curve of a parabolic null cylinder is a lightlike helix with $k_{L}=0$. Therefore, it is also an example of a null scroll whose mean curvature $H$ corresponds to the lightlike curvature of its base curve. For such null scrolls, since $H=k_{L}$, the Riccati equation (5) simplifies to the Bernoulli equation

$$
2 k_{1}^{\prime}+k_{1}^{2}=0 .
$$

By solving the equation (8), as a particular solution, we obtain $k_{1}=0$. Then frames $L$ and $\bar{L}$ coincide and a null scroll is a $B$-scroll. As the general solution, we obtain $k_{1}=\frac{2}{u}+c_{1}, c_{1} \in \mathbb{R}$, so we can construct the family of null scrolls whose mean curvature corresponds to the lightlike curvature of the base curve.

Theorem 3. For the given base curve with lightlike curvature $k_{L}$, the family of null scrolls whose mean curvature $H$ corresponds to $k_{L}$ is given by

$$
\mathcal{K}(c)=c(u)+v\left(\left(-\frac{2}{u^{2}}-k_{L}\right) \cdot c^{\prime}+\left(\frac{2}{u}+c_{1}\right) \cdot c^{\prime \prime}-c^{\prime \prime \prime}\right), c_{1} \in \mathbb{R} .
$$




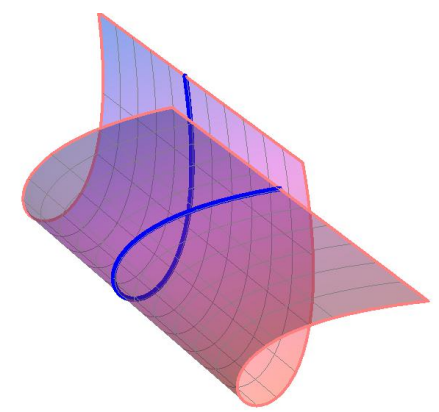

Figure 4: A parabolic null cylinder

Special case is the B-scroll given by

$$
f(u, v)=c(u)+v\left(k_{L} \cdot c^{\prime}-c^{\prime \prime \prime}\right) .
$$

Example 4. The null curve $c$ with parametrization

$$
c(u)=1 / 16\left(-4 u^{2}, u^{4}-4 \log u,-u^{4}-4 \log u\right)
$$

has the lightlike curvature $k_{L}=-3 /\left(2 u^{2}\right)$. The B-scroll with the base curve $c$ is given by (see Figure 5)

$f(u, v)=\frac{1}{16}\left(-4 u^{2}, u^{4}-4 \log u,-u^{4}-4 \log u\right)+v\left(\frac{3}{4 u}, \frac{7}{8 u^{3}}-\frac{15 u}{8}, \frac{7}{8 u^{3}}+\frac{15 u}{8}\right)$.

The family of null scrolls with base curve $c$ and the mean curvature $H=$ $-3 /\left(2 u^{2}\right)$ is given by

$\mathcal{K}(c)=\left\{c(u)+v\left(\frac{3-2 c_{1} u}{4 u}, \frac{6 c_{1} u^{5}+2 c_{1} u-7 u^{4}+15}{8 u^{3}}, \frac{-6 c_{1} u^{5}+2 c_{1} u+7 u^{4}+15}{8 u^{3}}\right)\right\}$,

where $c_{1} \in \mathbb{R}$ (see Figure 6).

\section{References}

[1] C. Baikoussis and T. Koufogiongos, Helicoidal surfaces with prescribed mean or Gaussian curvature, Journal of Geometry, 63(1)(1998), pp. 25-29.

[2] C. C. Beneki, G. Kaimakamis and B. J. Papantoniou, Helicoidal surfaces in three dimensional Minkowski space, Journal of Mathematical Analysis and Applications, 275 (2002), pp. 586-614. 


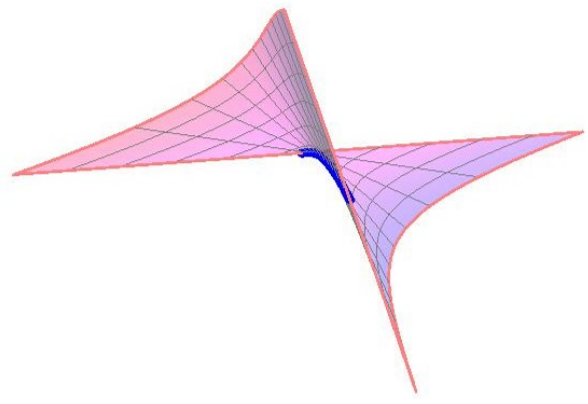

Figure 5: $B$-scroll with the base curve $c(u)=1 / 16\left(-4 u^{2}, u^{4}-4 \log u,-u^{4}-\right.$ $4 \log u$ )
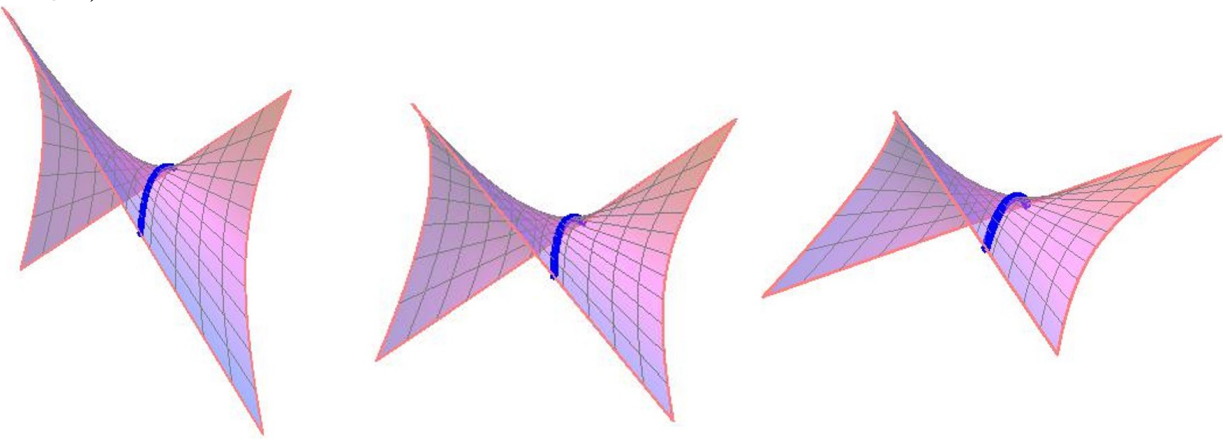

Figure 6: Examples of null scrolls with the base curve $c(u)=1 / 16\left(-4 u^{2}, u^{4}-\right.$ $\left.4 \log u,-u^{4}-4 \log u\right)$ and the mean curvature $H=-3 /\left(2 u^{2}\right)$

[3] S. Chanillo and M. Kiessling, Surfaces with prescribed Gauss curvature, Duke Mathematical Journal, 105(2) (2000), pp. 309-353.

[4] M. Do CARmo And M. Dajczer, Helicoidal surfaces with constant mean curvature, Tohoku Mathematical Journal, 34 (1982), pp. 425-435.

[5] K. L. Duggal and A. Bejancu, Lightlike Submanifolds of semiRiemannian Manifolds and Applications, Mathematics and its Applications, Kluwer Academic Publishers, Dordrecht, 1996.

[6] J. I. HAno AND K. Nomizu, Surfaces of revolution with constant mean curvature in Lorentz-Minkowski space, Tohoku Mathematical Journal, 36 (1984), pp. 427-437. 
[7] J. Inoguchi And S. LeE, Null curves in Minkowski 3-space, International Electronic Journal of Geometry, 1 (2008), pp. 40-83.

[8] K. Kenmotsu, Surfaces of revolution with prescribed mean curvature, Tohoku Mathematical Journal, 32 (1980), pp. 147-153.

[9] S. LeE And J. H. VARnAdo, Spacelike constant mean curvature surfaces of revolution in Minkowski 3-space, Differential Geometry - Dynamical Systems, 8 (2006), pp. 144-165.

[10] S. Lee And J. H. VARnado, Timelike surfaces of revolution with constant mean curvature in Minkowski 3-space, Differential Geometry - Dynamical Systems, 9 (2007), pp. 82-102.

[11] Ž. Milin Šipuš, LJ. Primorac GajČić And I. Protrka, Null scrolls as B-scrolls in Lorentz-Minkowski 3-space, Turkish Journal of Mathematics, 43(6) (2019), pp. 2908-2920, doi: 10.3906/mat-1904-140

[12] W. T. ReID, Riccati Differential Equations, New York: Academic Press, 1972 .

[13] J. TAFEL, Surfaces in $\mathbb{R}^{3}$ with prescribed curvature, Journal of Geometry and Physics, 17(4) (1995), pp. 381-390.

Željka MILIN ŠIPUŠ,

Department of Mathematics, Faculty of Science

University of Zagreb,

Bijenička cesta 30, 10000 Zagreb, Croatia.

Email: milin@math.hr

Ljiljana PRIMORAC GAJČIĆ,

Department of Mathematics,

University of Osijek,

Trg Ljudevita Gaja 6, Croatia.

Email: ljiljana.primorac@mathos.hr

Ivana PROTRKA

Faculty of Mining, Geology and Petroleum Engineering,

University of Zagreb,

Pierottijeva ul. 6, 10000 Zagreb, Croatia.

Email: ivana.protrka@rgn.unizg.hr 